\title{
Fluorescent Superlattices of Gold Nanoparticles: A New Class of Functional Materials
}

\author{
Edakkattuparambil Sidharth Shibu', Madathumpady Abubaker Habeeb Muhammed ${ }^{1}$, Keisaku Kimura ${ }^{2}$, and \\ Thalappil Pradeep ${ }^{1}(\bowtie)$ \\ ${ }^{1}$ DST Unit on Nanoscience (DST UNS), Department of Chemistry and Sophisticated Analytical Instrument Facility, Indian \\ Institute of Technology, Madras, Chennai 600 036, India \\ ${ }^{2}$ Graduate School of Material Science, University of Hyogo, 3-2-1 Koto, Kamigori-cho, Ako-gun Hyogo 678-1297, Japan \\ Received: 30 November 2008/Revised: 26 December 2008/ Accepted: 29 December 2008 \\ (C) Tsinghua University Press and Springer-Verlag 2009. This article is published with open access at Springerlink.com
}

\begin{abstract}
Fluorescent three-dimensional (3-D) superlattices of dansyl glutathione protected gold nanoparticles, with potential applications in molecular detection, have been synthesized at an air/water interface by controlling the $\mathrm{pH}$ of the nanoparticle suspension. The number of fluorophores per nanoparticle was calculated to be 127. Morphologies of the superlattice crystals were examined using scanning electron microscopy (SEM). Most of the crystals observed were triangular in shape. High-resolution transmission electron microscopy (HRTEM) and small angle X-ray scattering (SAXS) were used to study the packing of nanoparticles in these crystals. Both these studies showed that the nanoparticles were arranged in a face-centered cubic (fcc) pattern with a particle-particle distance (center-center) of $\sim 10.5 \mathrm{~nm}$. Evolution of the crystal morphologies with time was also examined. The fluorescence properties of these triangles were studied using confocal fluorescence imaging and confocal Raman mapping, which were in good agreement with the morphologies observed by SEM. The superlattice exhibits near-infrared (NIR) absorption in the range 1100-2500 nm. Easy synthesis of such functional nanoparticle-based solids makes it possible to use them in novel applications. We utilized the fluorescence of dansyl glutathione gold superlattice crystals for the selective detection of bovine serum albumin (BSA), the major protein constituent of blood plasma, based on the selective binding of the naphthalene ring of the dansyl moiety with site I of BSA.
\end{abstract}

\section{KEYWORDS}

Periodic self-assembly, dansyl glutathione, fluorescent superlattice, confocal fluorescence imaging, selective binding

\section{Introduction}

Fabrication of nanoparticle structures with well defined two- or three-dimensional (2-D or 3-D) configurations called, "artificial solids" [1] is important from many perspectives. Hierarchical self-assembly of nanoparticles into a 2-D or 3-D superlattice facilitates manipulation of the structures with enhanced and tunable quantum confinement effects which opens up the possibilities of developing new classes of nanomaterials and devices with potential applications in light emitting diodes [2],

Address correspondence to pradeep@iitm.ac.in 
solar cells [3], molecular electronic architectures [4], high-density data storage [5], catalysis [6], biochemical sensors [7, 8], biocompatible materials [9], and so on. The tremendous interest in particle superlattices is also driven by their already established applications in various fields resulting from the collective properties of the building blocks, which help us to understand electronic effects such as the metal-insulator transition and changes in magnetic and optical properties [10, 11]. One could possibly control the assembly of such organized solids, which opens up new opportunities for fundamental studies as well as engineering of advanced materials. Moreover, the crystal structure of nanoparticle superlattices is strongly affected by the geometrical shape of the adjacent nanoparticles. Packing is determined to a large extent by geometrical considerations [12-16]. One of the most straightforward approaches for the construction of 2-D or 3-D superlattices currently being exploited is the bottom-up assembly technique, which includes electrostatic self-assembly of oppositely charged nanoparticles with narrow size distribution [17], evaporation of the solvent on a substrate [1, 18-29], self-organization of nanoparticles at interfaces [3033], crystallization of nanoparticles from nanoparticle solutions by precipitation or sedimentation [10, 34], wet deposition by supramolecular interactions between the nanoparticles and a surface [35-37], and self-assembly of nanoparticles into microdimensional structures through hydrogen bonding [38-43]. Recently, binary nanoparticle superlattices based on metallic [44], or a combination of metallic and semiconductor building blocks were reported [24, 45]. Binary superlattices combining $\mathrm{PbTe}$ and $\mathrm{Ag}_{2} \mathrm{Te}$ [46], and CdSe and CdTe nanocrystals (NC) $[47,48]$ have also been reported recently.

In most of the earlier reports of superlattices, the properties of the molecular coating of the nanoparticles were not focused upon. In several of those cases, the monolayer coatings were simple alkyl chains or their variants. We undertook a program recently to explore the properties of functional nanoparticle superlattices, in which the protecting monolayers have functional attributes. Fluorescence is one such property which could be useful for a variety of reasons, especially when it is coupled with the properties of nanoparticle assembly. In this context, we recently reported the self-assembly of a fluorescein-based dye conjugated mercaptosuccinic acid (5-((2-(and-3)-S(acetylmercapto)-succinoyl)amino)fluorescein or SAMSA) nanoparticles into 3-D crystals by utilizing hydrogen bonding [43]. The SAMSA molecules were anchored on the surface of mercaptosuccinic acid (MSA) capped nanoparticles by a ligand exchange reaction and the resulting fluorescent nanoparticles self-organized into 3-D superlattices. It was found that around six fluorescent molecules were bound on each nanoparticle. The presence of fluorophores in the superlattice was confirmed by the Raman images and the fluorescence spectrum collected from a single crystal.

In the present work, we utilize a comparatively cheap and easily synthesizable flurophore, dansyl glutathione and perform detailed structural and spectroscopic characterization of such fluorescent particle crystals by an array of techniques. The dansyl [5-(dimethylamino)naphthalene-1-sulfonyl] moiety exhibits green fluorescence when conjugated with any aliphatic or aromatic primary amine; such molecules are known as "environment-sensitive fluorophores". Moreover, the dansyl group is widely used as a fluorescent label in immunofluorescence methods, yielding fluorescent $\mathrm{N}$-terminal amino acids and peptide derivatives. In this paper, we report the formation of 3-D superlattices of dansyl glutathione capped nanoparticles by hydrogen bonding of the carboxylic group present in the glutathione. The fluorescent nanoparticles were allowed to grow at an air/water interface at highly acidic $\mathrm{pH}(<1)$ and after aging of the crystals, they were carefully transferred to carbon coated grids and Si-wafer/ conducting glass surfaces for high-resolution transmission electron microscopy (HRTEM) and field emission scanning electron microscopy (FESEM) measurements. These 3-D crystals were characterized by various microscopic and spectroscopic techniques to derive detailed structural information. FESEM measurements revealed the presence of different morphologies in the superlattice films, including triangles, icosahedra, triangular pyramids, and 
diamond-like structures. Of these different geometries, we mainly focused on two different types of triangles. As an example of their applications, we demonstrate their use in the detection of bovine serum albumin (BSA). These systems can be considered as a new class of functional materials and may be used for different applications depending on the functionality introduced.

\section{Results and discussion}

The number of dansyl glutathionate (-SGD) ligands present per nanoparticle was calculated to be $\sim 127$. This calculation followed the methodology described in Ref. [43], using the extinction coefficient of the ligand. This is based on the fact that the difference in the concentration of -SGD ligands in the solution before and after the reaction gives the number of -SGD ligands on the nanoparticles. Parent dansyl glutathione (DGSH) and -SGD protected nanoparticles were characterized by $\mathrm{UV} /$ vis and fluorescence spectroscopy, ESI-MS and HRTEM. Figure 1(a) shows the $\mathrm{UV} /$ vis absorption spectra of DGSH (b) and $\mathrm{Au}$ nanoparticles protected with $N$-acetylglutathionate (-SGAN) and -SGD groups, denoted Au@SGAN / SGD (a). The weak surface plasmon of Au@SGAN/SGD around 530 nm clearly indicates the presence of $3^{-}$ $4 \mathrm{~nm}$ diameter particles. DGSH itself shows two sharp absorption features in the UV region (at $286 \mathrm{~nm}$ and $330 \mathrm{~nm}$ ), corresponding to the allowed $\pi-\pi^{*}$ and forbidden $n-\pi^{*}$ electronic transitions, respectively. The same absorption peaks were also observed for Au@ SGAN/SGD nanoparticles, albeit with weak intensities. Figure 1(b) shows the excitation and emission spectra of DGSH (c, d) and Au@SGAN/SGD $(\mathbf{e}, \mathbf{f})$. The spectra are similar, but the positions of the peak maxima are slightly different. ESI-MS (-ve mode) of Au@SGAN/SGD nanoparticles shows two peaks at $m / z 539$ and 234 which correspond to DGS- and the dansyl group respectively (Fig.1(c)). Figure 1(d) shows the ${ }^{1} \mathrm{H}$ NMR spectrum of Au@SGAN/SGD nanoparticles, showing the peaks due to the glutathione moiety in the 2.0 $-4.5 \mathrm{ppm}$ region. These resonances are somewhat broad in nature as the glutathione moieties are very close to the nanoparticle surface [49]. The peaks at 2.2 (e), 2.5 (d), and 3.7 (f) ppm respectively correspond to the $\gamma-\mathrm{CH}_{2}, \beta-\mathrm{CH}_{2}$, and $\alpha-\mathrm{CH}$ of the glutamic acid group in the tripeptide [50,51]. The peak at 3.6 (a, b) ppm is due to the overlap of the glycine $\alpha-\mathrm{CH}_{2}$ group and the cysteine $\alpha-\mathrm{CH}$ group [50]. The peak corresponding to the $\mathrm{\beta}-\mathrm{CH}_{2}$ of cysteine (c) expected at ca. 3 ppm [50] has almost completely disappeared since these protons are very close to the gold surface. The NMR spectrum of $\mathrm{Au}_{25}$ clusters partially protected with -SGAN was reported recently [50]. The positions and peak shapes observed here are similar. The peak at $2.2 \mathrm{ppm}(\mathrm{h})$ is due to the acetyl $-\mathrm{CH}_{3}$ protons (-SGAN) and overlaps with $\gamma-\mathrm{CH}_{2}$ of glutamic acid (e). The peak at $2.8 \mathrm{ppm}(\mathrm{g})$ is

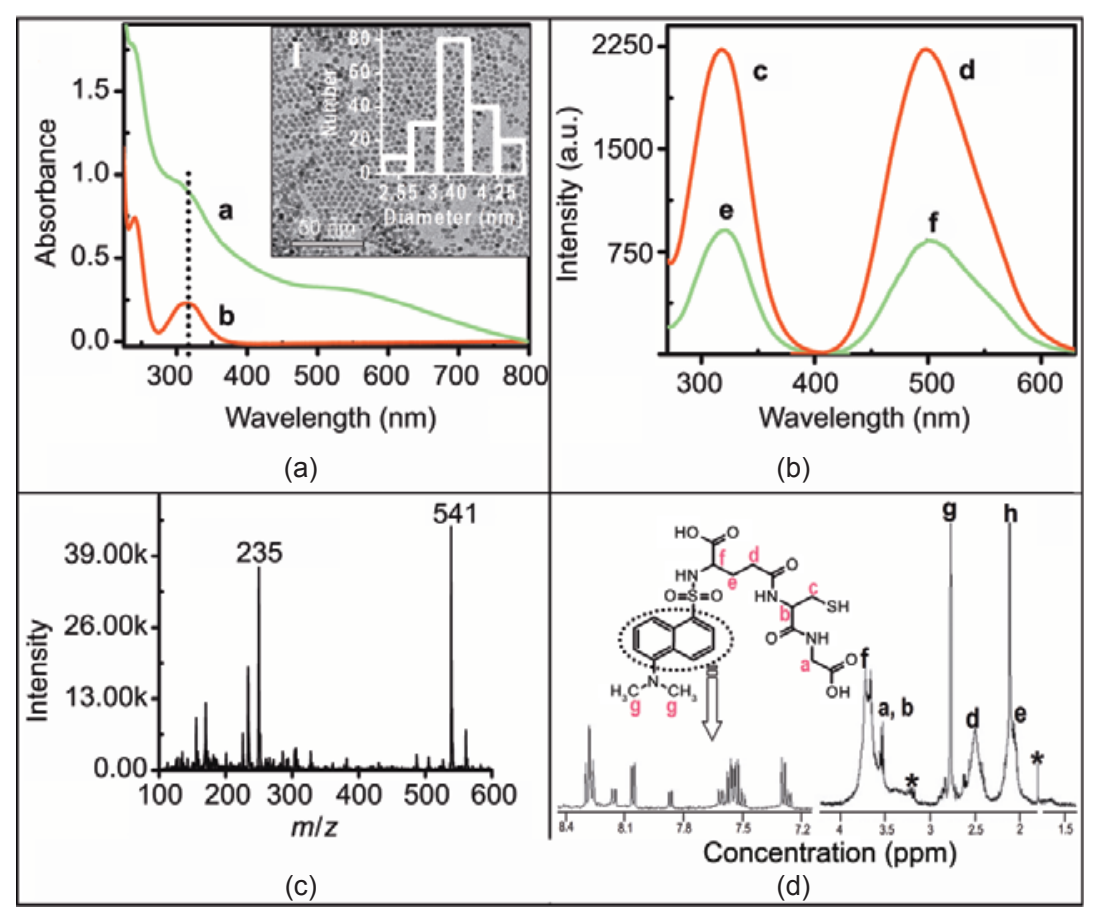

Figure 1 (a) UV/vis spectra of (a) Au@SGAN/SGD and (b) DGSH. Inset I in (a) shows the HRTEM image of Au@SGAN/SGD nanoparticles; the average particle size was calculated to be around $3.5 \mathrm{~nm}$, shown by the histogram. (b) Excitation and emission spectra of DGSH (c, d) and Au@SGAN/SGD (e, f). (c) ESI-MS (-ve mode) of Au@SGAN/SGD showing peaks at m/z 234 and 539. (d) ${ }^{1} H$ NMR spectrum of Au@SGAN/SGD nanoparticles; the aromatic peaks (marked with the arrow) are not broadened. Peak assignments are indicated on the molecular structure for DGSH. The peak labeled $\mathbf{h}$ is due to acetyl $-\mathrm{CH}_{3}$ protons of - SGAN. Peaks labeled * are spikes 
due to the two $-\mathrm{CH}_{3}$ groups of the dansyl moiety. Peaks in the range 6.0-8.2 ppm correspond to the aromatic region of the dansyl moiety [51]. These peaks are not broadened, since the dansyl group is far away from the metal surface. The spectral data confirm the presence of dansyl glutathione groups on the nanoparticle surfaces. The average core diameter of the Au@SGAN/SGD nanoparticles was found to be $\sim 3.5 \mathrm{~nm}$. Inset I in Fig. 1 shows the HRTEM image and corresponding histogram of the nanoparticles. In a typical nanoparticle of this size, it is estimated that $\sim 196$ thiolate species are required for complete coverage. Thus, the presence of $\sim 127$ dansyl glutathione means that $\sim 69$-SGAN groups are present. This corresponds to an -SGD:-SGAN ratio of 1.85 , close to that used in the synthesis (2.33). Thus, the starting composition is reflected in the final product.

The morphology of the superlattices was examined in detail by FESEM. We carefully lifted the superlattice film by using a copper wire loop and placed it on a polished silicon wafer or clean conducting glass. The samples were carefully washed with ethanol and left to dry for a few hours in the ambient air. The dried samples were mounted on the SEM stub and conduction between the sample and the stub was facilitated with a conducting carbon tape. All the SEM measurements were carried out at $30 \mathrm{kV}$. Figure 2(a) shows a large area FESEM image of the gold nanoparticle superlattices. The image shows that most of them are "triangular" in shape (marked by circles).

In addition to triangles, a few other shapes such as icosahedra, triangular pyramids, diamond-like structures, etc. were also observed and these are shown in the Electronic Supplementary Material (ESM) (Fig. S-1). In all such superlattices, triangular morphologies are the most common. It appears that the low energy of the (111) planes and their abundance in triangular structures is the reason for this preference in morphology. SEM and X-ray diffraction confirm the preferential occurrence of (111) planes. Upon closer examination, we see two types of triangles and each one is expanded in images (Figs. 2(b) and 2(c). The main difference between these two triangles is in the way of stacking nanoparticles. In the case of triangle 1, which is more common (Fig. 2(b)),

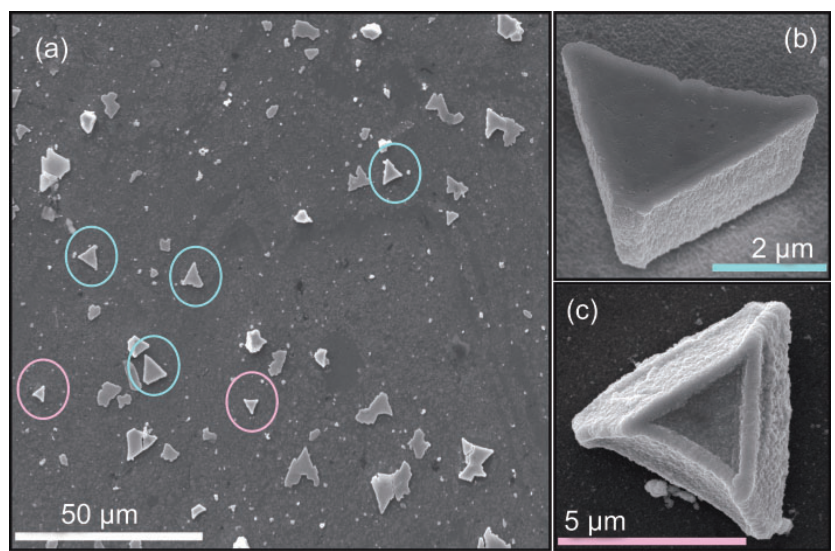

Figure 2 (a) Large area FESEM image of the gold nanoparticle superlattices. Different types of "triangles" are marked by circles and are color coded differently. (b) and (c) show the two different types of triangles observed

the morphology is similar to a prism and its surface is almost flat, whereas in case of triangle 2 (Fig. 2(c)) it is like a pyramid with trench on top of the crystal. On the top of the crystal, the nanoparticles are arranged in such a manner as to form a thick edge.

Magnified images of the superlattices show a regular arrangement of the particles throughout the crystal. Two of these images are shown in Fig. 3. Figure 3(a) is a large area image taken from an edge of a crystal. The inter-particle distance was found to be around $10.6 \mathrm{~nm}$, consistent with the HRTEM and small angle X-ray scattering (SAXS) measurements; details of these are given later. Figure $3(b)$ is a large area image taken from the surface of the crystal showing the regular arrangement of nanoparticles. The interparticle spacing was found to be $10.6 \mathrm{~nm}$ in this case also. Another magnified image of the surface of a superlattice crystal, with improved contrast

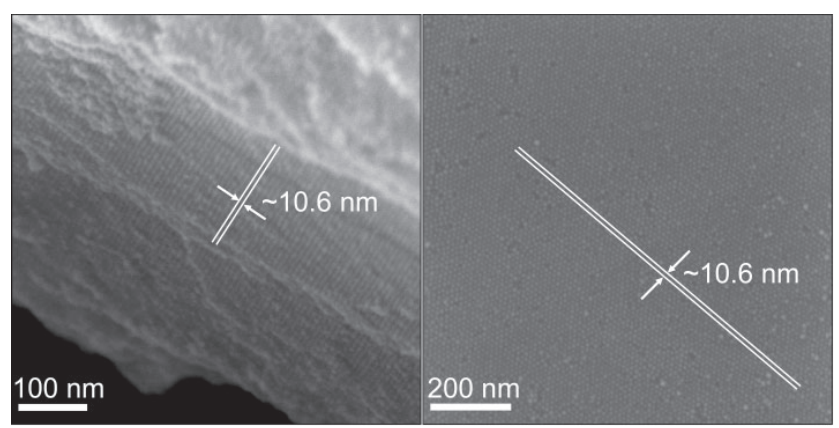

(a)

(b)

Figure 3 High-resolution FESEM images of the superlattice triangles showing particle arrangements on an edge (a) and the top surface (b) of a prism 
allowing defects to be observed, is given in the ESM (Fig. S-2). Details of the nanoparticle assembly can be seen in all these images.

Stacking of the nanoparticles in the 3-D superlattices was characterized by SAXS. The superlattice samples were transferred to a thin mica sheet and dried under ambient conditions. As SAXS is a bulk measurement, the data refer to a combination of different types of superlattice morphologies. After the measurements, the contribution of the mica background was subtracted. The data were measured over $120 \mathrm{~min}$ for each sample. Figure 4 (a) shows the a plot of the background-corrected intensity vs scattering vector, $q=4 \pi \sin \theta / \lambda$, where $\theta$ is the scattering angle and $\lambda$ is the $X$-ray wavelength, for the Au superlattices. Figure 4(b) shows a plot of intensity vs $2 \theta$ data obtained after background and baseline corrections. We can see that the constituent gold nanoparticles are stacked in a (face-centered cubic) pattern rather than hexagonal close-packed (hcp) in the 3-D array, with a (111) spacing (particle-particle) of 10.5 $\mathrm{nm}$ as determined by the simulated peak positions. The spacing between (220) planes was also calculated and is around $4.9 \mathrm{~nm}$. All these lattice parameters are in good agreement with the HRTEM and SEM data. The various diffraction planes are labeled in Fig. 4(b).

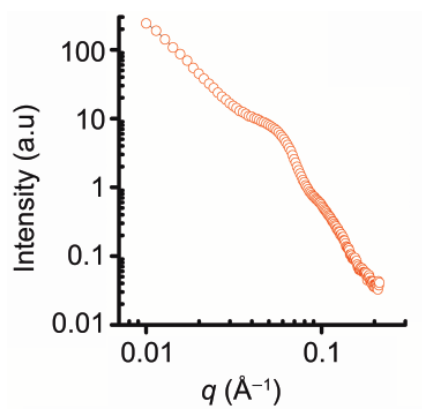

(a)

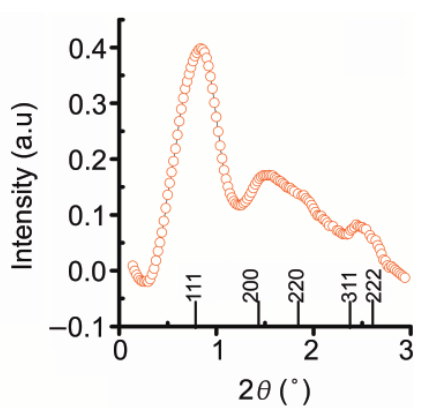

(b)
Figure 4 (a) Plot of background-corrected intensity vs scattering vector $(q=4 \pi \sin \theta / \lambda)$ of Au@SGAN/SGD superlattices; (b) plot of intensity vs $2 \theta$ data obtained after background and baseline corrections. The indexing is done for an fcc unit cell

To understand the details of the particle arrangement in the superlattice crystals, we analyzed the films by HRTEM. Figure 5(a) shows a TEM image of the superlattice. Due to the thickness of the crystal, we could analyze the particle arrangement only at the edge of the triangle and inset I shows a low magnification image of an edge of a superlattice triangle. Inset II in Fig. 5(a) shows a high magnification image of a single nanoparticle. The particle shows a truncated octahedral (TO) shape with $\{100\}$ and $\{111\}$ facets. Figure $5(b)$ is a large area image showing that the particles adopt a regular and periodic arrangement. In the image, a $[110]_{\text {SL }}$ projection of the unit cell of the superlattice is represented by a rectangular box, where the subscript SL refers to the superlattice. In this image, the bright spots between the nanocrystals correspond to the lower electron density channels of the branched adsorbed molecules. Figure 5(b) reveals that no

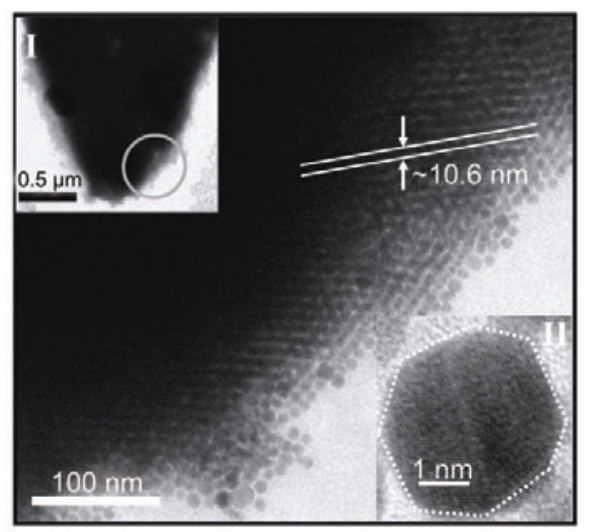

(a)

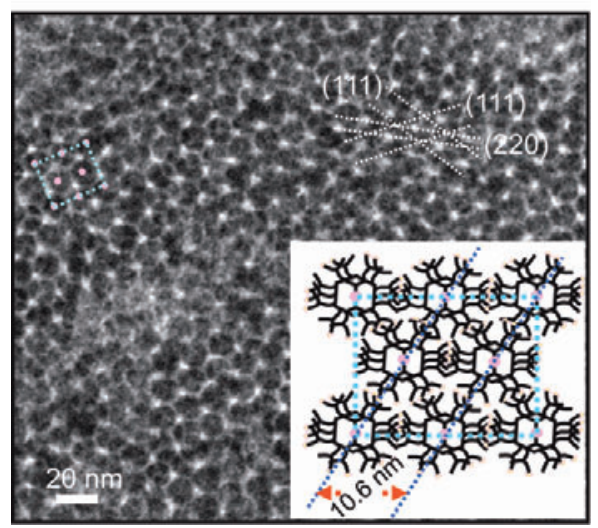

(b)

Figure 5 (a) TEM image of the Au@SGAN/SGD nanoparticle superlattice. Inset I shows a low magnification image of one edge of a superlattice crystal and the edge expanded in (a) is marked with a circle. Inset II shows a high magnification image of a single nanoparticle used for the synthesis of superlattice crystals which has a TO shape with $\{100\}$ and $\{111\}$ facets. (b) Magnified TEM image of the superlattice which reveals no further growth of nanoparticles. Highly organized assembly of nanoparticles over a large region is seen. Inset in Fig. 5(b) is a model showing the face-to-face assembly of the nanocrystals, which is in good agreement with the image shown in Fig. 5(b) 
further growth of nanoparticles occurs in the superlattice crystal. The interplanar spacings for (111) and (220) planes are around 10.6 and $5.0 \mathrm{~nm}$, respectively, which are in good agreement with the SAXS data. Inset in Fig. 5(b) is a model which shows the face-to-face assembly of the nanocrystals with strong inter-chain interactions. The particle size is the same as that shown in Fig. 1. The inter-particle distance is in agreement with the core dimension $(3.5 \mathrm{~nm})$ and the length of the monolayer $(2.65 \mathrm{~nm})$. A cubic arrangement of such monolayer-protected particles with close contact of these spheres would lead to an inter-particle distance of $8.8 \mathrm{~nm}$. The increase in the distance can be accounted for in terms of the conformational freedom of the dansyl moiety and the peptide chain. The TEM image is in accordance with that presented by Wang et at. [52, 53], with the only difference being that the spots show bright contrast which is due to the difference in the type of alkyl chains forming the assembly. The carboxylic acid groups of these monolayers form cyclic arrangements of hydrogen bonds with and without the mediation of water molecules, imparting stability to the nanoparticle assembly [38 $-43]$. It is necessary to have undissociated carboxylic acid groups to facilitate hydrogen bonds, justifying the acidic $\mathrm{pH}$ used in the synthesis.

In order to study the spatial distribution of gold in the superlattice film, elemental mapping of a single crystal was carried out using energy dispersive analysis of X-rays (EDAX). Figure S-3(a) (ESM) shows the EDAX spectrum collected from the superlattice triangle 1 (Fig. S-3(b)). The EDAX maps of $\mathrm{Au} \mathrm{M} \alpha, \mathrm{Au} \mathrm{L} \alpha$, and $S \mathrm{~K} \alpha$ of triangle 1 are also given the ESM (Figs. S-3(c), S-3(d), and S-3(e)). The table (inset) in Fig. S-3 shows the corresponding elemental composition of the superlattice crystals, showing $92.85 \mathrm{wt} \%$ gold. Tilted images of the crystals at different angles of $0^{\circ}, 20^{\circ}, 40^{\circ}$, and $60^{\circ}$ were measured to determine the thickness of the crystals (ESM, Fig. S-4). The thickness of a normal triangle is around $1.22 \mu \mathrm{m}$. Triangle 2 also showed similar EDAX maps.

In order to understand the growth process of the complicated structure of triangle 2, we conducted a time-dependent crystal growth study using SEM. For this, the $\mathrm{pH}$ of the samples was adjusted and they were left in different $6 \mathrm{~mL}$ bottles. Although the crystallization started in the first week, the first sets of measurements were only done during the second week. We transferred the samples every two weeks, up to two months (from separate bottles) and completed the measurements. SEM images (1-4) of crystals collected at different stages of growth are given in Fig. 6, showing the formation of a trench in the middle. The arrangement of nanoparticles which results in the thick upper edge and the thin lower edge may be inferred from this morphology. The crystal growth was completed over a period of two months and we could not see any more structural changes after this period. The spatial distribution of gold and sulfur was confirmed using EDAX. Elemental maps of gold and sulfur were recorded for all the samples (Fig. 6).

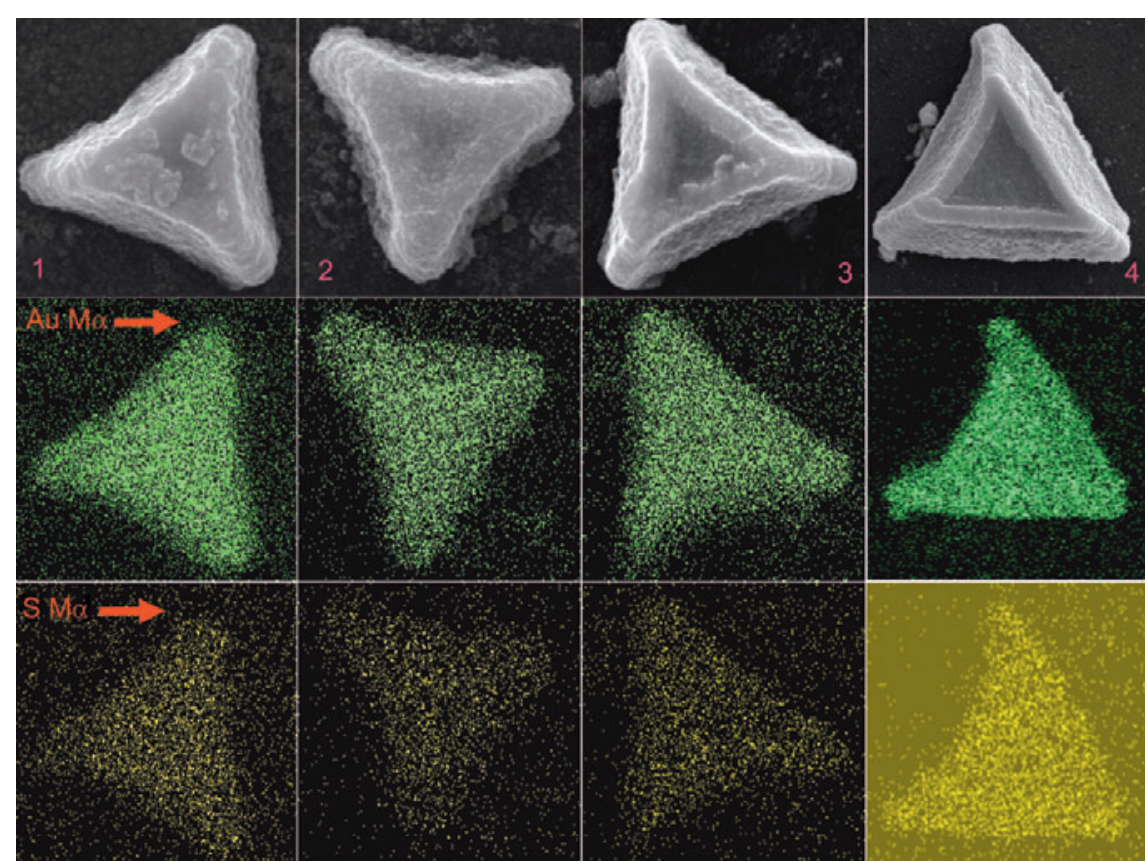

Figure 6 The time dependent SEM images of the morphology of triangle 2 (see Fig. 2(c)) at different time intervals $(2,4,6$, and 8 weeks). Elemental mappings based on $\mathrm{Au} \mathrm{M \alpha}$ and S Ka at all four stages are shown. The images $1,2,3$, and 4 correspond to edge lengths of $4,5,6$, and $10 \mu \mathrm{m}$, respectively 
Interesting images of these superlattice triangles were collected by confocal fluorescence, which are in good agreement with the FESEM data. The fluorescence measurements were done using a confocal microscope with a $458 \mathrm{~nm}$ laser, which is the shortest wavelength available in the microscope. The fluorescence images of superlattice triangles were collected by integrating the intensities in the 500$650 \mathrm{~nm}$ window where the emission from the superlattice occurs. The superlattice film was transferred to a thin glass plate and dried under ambient conditions. The sample plate was mounted on the piezoequipped scan stage and directly imaged using the laser scanning confocal microscope. Figure 7 shows the confocal fluorescence images and corresponding overlay structures collected from triangle 2 at different depths (z-scan). The most striking feature of the fluorescence image of this type of crystal is the difference

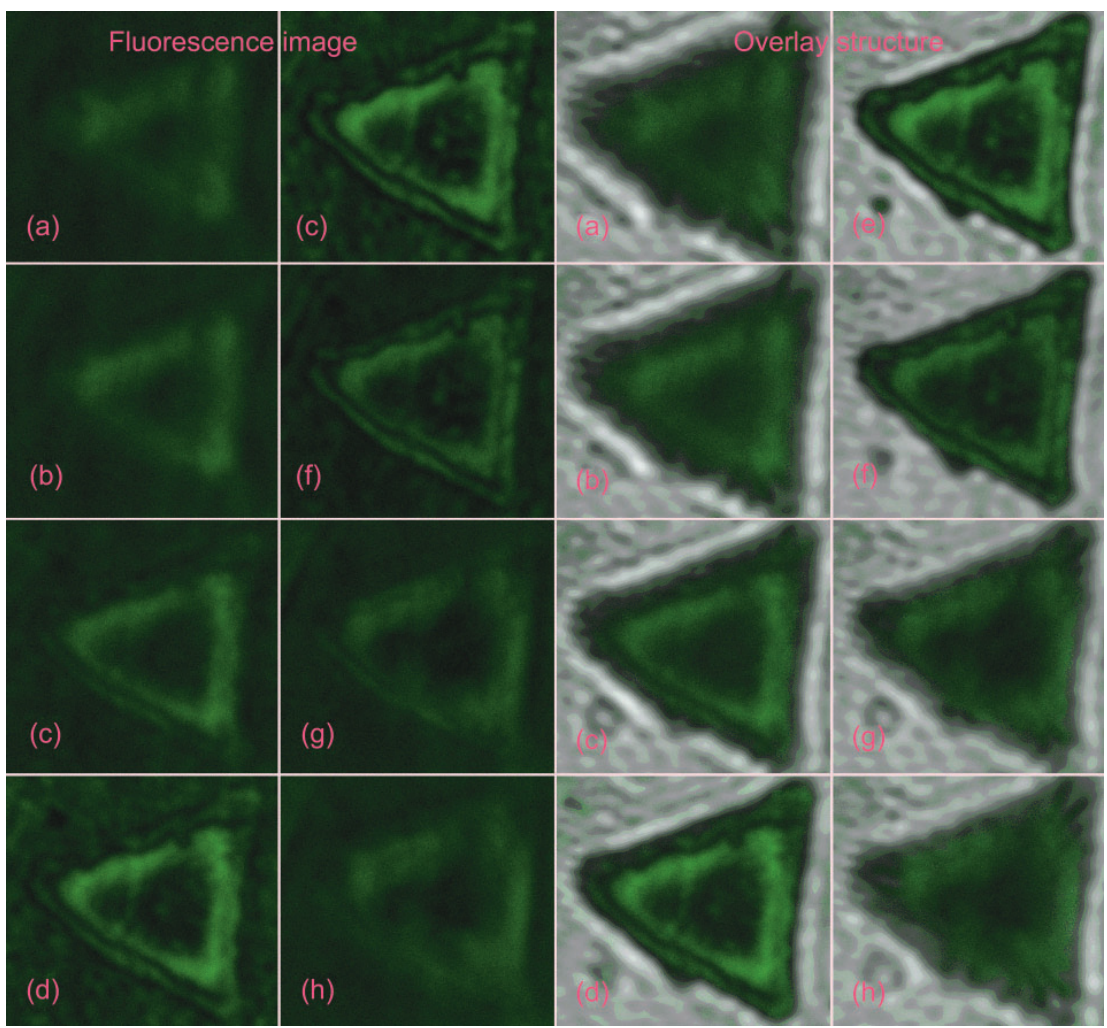

Figure 7 The confocal fluorescence images (z-scan) and corresponding overlay structures collected from triangle 2 (see Fig. 2(c)) of the superlattice crystals excited by a $458 \mathrm{~nm}$ laser, which was the shortest wavelength available in the microscope. In the images, the inner top edge of the triangle is thick which emits more light whereas the outer bottom edge is thin which emits less light. The lack of fluorescent nanoparticles in the middle trench created a dark triangular portion (a) to ( $h$ ) are from top to bottom with the top of the crystal coming into focus at (d) in the fluorescence intensity at the edges. Here we can see one inner thick triangle with maximum fluorescence intensity and one outer thin triangle with minimum fluorescence intensity and a middle trench with zero intensity. This is consistent with the difference in the thickness of the inner and outer edges of triangle 2, as we have seen in the SEM image (Fig. 2(c)). Since the inner top edge of the triangle is thick, this emits more light while the outer bottom edge is thin which emits less light. The lack of fluorescent nanoparticles in the middle trench creates a dark triangular portion. We also collected the fluorescence images from triangle 1 and the images are comparable with the SEM images. In this case, we observed uniform fluorescence over all parts of the crystal. This can be explained by the fact that the crystal is flat as we saw in the SEM. The confocal fluorescence images and corresponding overlay structures collected at different depths (z-scan) are given in the ESM (Fig. S-5). Although number density is attributed to the difference in fluorescence intensity of triangle 2, a contribution due to enhanced emission from the edges due to higher electromagnetic fields cannot be ruled out.

The fluorescent nature of the superlattice crystals was confirmed by imaging the crystals using confocal Raman microscopy. The samples were transferred to a thin glass plate and mounted on a piezo-equipped scan stage to enable spectral imaging. Figure 8 shows the confocal fluorescence images (z-scan) of the triangle 1 at different depths, Figs. 8(a)-(d). Figure $8(\mathrm{a})$ is the fluorescence image of a crystal collected at the focal plane which shows maximum fluorescence intensity. Figure $8(\mathrm{~b})$ is the corresponding image collected at $0.5 \mu \mathrm{m}$ below the initial focal plane which shows fluorescence intensity lower than the first case. We also collected the fluorescence images at $1 \mu \mathrm{m}$ and $1.5 \mu \mathrm{m}$ below the focal plane and the same trend was 


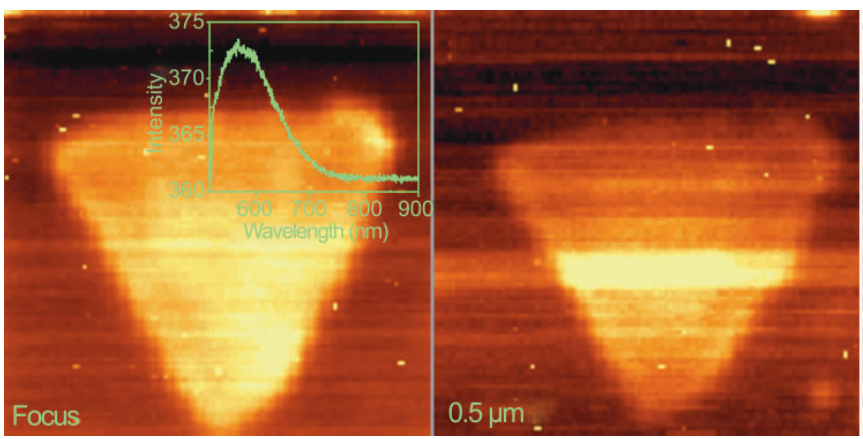

(a)

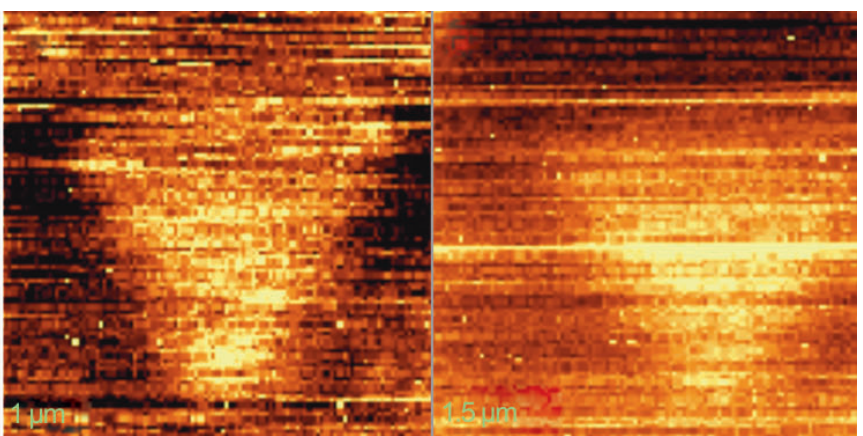

(c)

(d)

Figure 8 Confocal fluorescence images (a)-(d) of the triangle collected at different depths (at focus, and $0.5 \mu \mathrm{m}, 1 \mu \mathrm{m}$, and $1.5 \mu \mathrm{m}$ away from the focus). Inset in (a) shows the corresponding emission collected from the triangle. The image does not represent the true color of the fluorescence

observed in both cases. The inset in Fig. 8(a) shows the corresponding emission spectrum collected from the triangle with an emission maximum of 590 $\mathrm{nm}$. The red shift in the emission maximum can be attributed to the large electronic interaction of the fluorophore and the nanoparticles in the 3-D solids. The optical image of a single triangle was also taken under white light illumination and is given in the ESM (Fig. S-6).

Figure S-7 in the ESM shows the contact mode atomic force microscopy (AFM) image (20 $\mu \mathrm{m} \times$ $20 \mu \mathrm{m}$ ) of the superlattice triangle 1 with an average edge length of $7.5 \mu \mathrm{m}$ and average thickness of 0.54 $\mu \mathrm{m}$. The edge length of the crystal was measured from the height profile analysis. The inset shows the corresponding height profile of the crystal along the dashed line in Fig. S-7. As can be seen from the SEM, TEM and AFM images, the sides of the crystals are not sharp and show corrugations at the nanometer scale. As the morphology is clear from the SEM images, AFM was not attempted on triangle 2.

Detection of biomolecules using gold nanoparticles has been attracting considerable interest over the past few years [54]. We utilized the fluorescence of the dansyl moiety for the specific detection of bovine serum albumin (BSA). Serum albumin is the major protein constituent of blood plasma. Specific delivery of ligands by BSA is considered to be due to the presence of two selective binding sites, namely site I and site II [55]. Dansyl amides are known to bind with site I through hydrophobic interactions [55]. We transferred the Au@SGAN/SGD superlattice film onto a quartz disc and dried it at room temperature.
The sample was excited at $330 \mathrm{~nm}$ and emission was collected at $587 \mathrm{~nm}$, where there is no emission from BSA. After this, $10 \mu \mathrm{L}$ BSA solution $(1 \mathrm{nmol} / \mathrm{L})$ in phosphate buffer was dropped on the film and emission was collected using the same excitation wavelength. As expected, we observed emission spectra with greater intensity compared to the blank superlattice film. A further enhancement with a bathochromic shift from $587 \mathrm{~nm}$ to $592 \mathrm{~nm}$ was observed with higher concentrations ( $\mu \mathrm{mol} / \mathrm{L}$ and $\mathrm{mmol} / \mathrm{L}$ ) of BSA ((a)-(d)). This rapid fluorescence enhancement of the dansyl fluorophore is attributed to the specific binding of the naphthalene ring of the dansyl moiety with site I of BSA. Figure 9(a) shows the corresponding fluorescence spectra during the fluorimetric titration experiment. A schematic representation of the specific binding of the naphthalene ring with BSA is shown in Fig. 9(b). While a free nanoparticle is also expected to show such a change, experiments in the solid state on a superlattice have advantages in terms of increased stability, the possibility of observation using optical microscopy, and incorporation on biochips enabling simultaneous Raman measurements.

We measured the UV/vis/near-infrared (NIR) absorption spectrum of the superlattice films. Measurements were done by depositing the films on glass plates and the contribution of the glass plate was subtracted. For the superlattice films, in addition to the nanoparticle absorption at $545 \mathrm{~nm}$, a new broad absorption peak was observed in the 1100-2500 $\mathrm{nm}$ window. The UV/vis/NIR absorption spectrum of the Au@SGAN/SGD nanoparticle superlattices is 


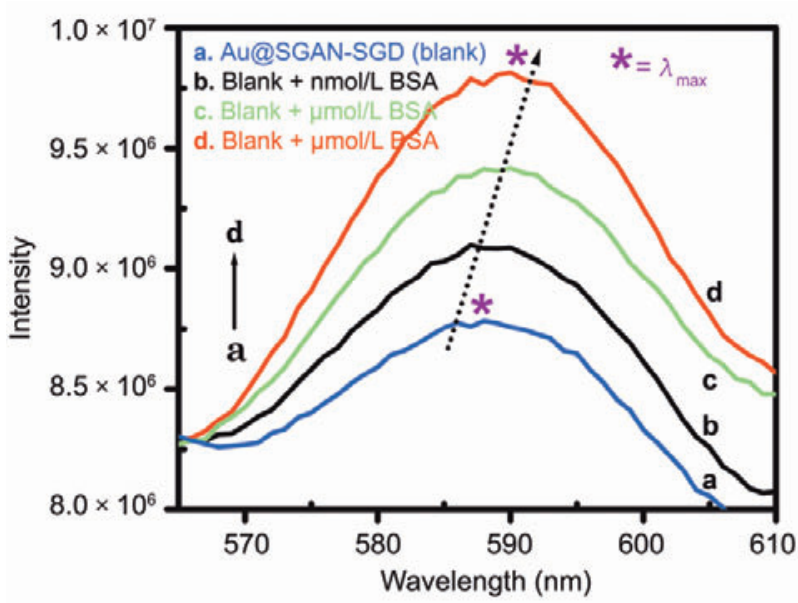

(a)

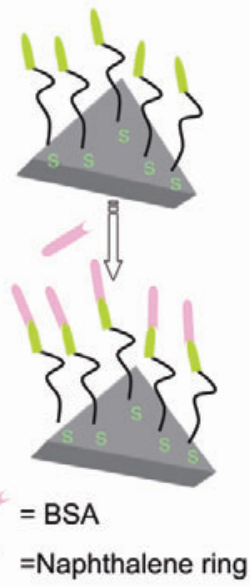

(b)
Figure 9 (a) Fluorescence spectra of Au@SGAN/SGD superlattice film during the course of fluorimetic titration with different concentrations of BSA; (a) blank, (b) nmol/L, (c) $\mu \mathrm{mol} / \mathrm{L}$ and (d) $\mathrm{mmol} / \mathrm{L}$. The excitation wavelength was $330 \mathrm{~nm}$. The spectra show a bathochromic shift from $587 \mathrm{~nm}$ to $592 \mathrm{~nm}$. (b) Schematic representation of the specific binding of the naphthalene ring with BSA

given in the ESM (Fig. S-8). Superlattice formation is facilitated by weak interactions such as hydrogen bonding between neighboring nanoparticles. These interactions occur when nanoparticles are placed in proximity, and result in new electronic and optical properties unique to the solid state. When the interparticle distance decreases, coupling between the particles increases which will consequently increase the energy bandwidth of the nanoparticle superlattices. In metal nanoparticle superlattices prepared on a Langmuir trough, an insulator to metal transition was observed when data were measured as a function of monolayer chain length $[1,19]$. This is due to the exchange interactions found when decreasing $D / 2 R$, where $D$ is the distance between nanoparticle centers and $R$ is the nanoparticle radius. Significant coupling is observed when $D / 2 R \approx 1.2$, where an insulator to metal transition occurs. In case of the Au@SGAN/SGD nanoparticle superlattice, the inter-particle distance is $10.6 \mathrm{~nm}$ and $D / 2 R$ is ca. 1.5. In this limit, strong exchange interactions producing an infrared absorption may not occur [19]. Apart from the insulator to metal transition, one possible reason for the infrared absorption could be the collective interplasmon coupling of nanoparticles in the 3-D array [19]. NIR absorption in superlattices due to the coupling of surface plasmon resonance has been reported by Huang and co-workers recently [56].
We are currently not certain about the nature of the absorption in our materials, however.

The creation of crystals with various morphologies is indeed interesting. Although hexagonal symmetry is observed in the packing, five-fold symmetric structures such as icosahedra, decahedra, and pentagonal rods were also formed during the crystal growth. Such structures have been previously reported in superlattices [42]. As fivefold symmetry cannot give a space filling structure, angular strains are introduced as the crystal dimensions increase. Relaxation of the strain occurs by reduction in surface energy of the crystal due to the presence of lower surface energy facets such as (111), as seen in Fig. S-1(c) in the ESM. The interfacial growth of crystals produces triangular shapes more often than other structures, whereas the restriction imposed by interfacial growth results in morphologies such as triangle 2. In this case, it appears that the diffusion of nanoparticles into the growing crystal occurs to a greater extent from the sides which may result in a depression in the middle as the size gets larger.

\section{Experimental}

\subsection{Materials}

All chemicals were commercially available and used without further purification. $\mathrm{HAuCl}_{4} \cdot 3 \mathrm{H}_{2} \mathrm{O}$, methanol (GR grade), ethanol (GR grade), and glutathione, (GSH, $\gamma$-Glu-Cys-Gly, MW =307) were purchased from SRL Chemical Co. Ltd, India. $\mathrm{NaBH}_{4}(>90 \%)$ was purchased from Sigma Aldrich. Dansyl chloride, ( $\mathrm{DnCl}, 5$-dimethylamino1-naphthalenesulfonyl chloride, $\mathrm{MW}=270$ ), and dithiotreitol, (DTT, 1, 4-bis-sulfanylbutane-2, 3-diol, $\mathrm{MW}=154$ ) were purchased from Acros Organics. Acylation of GSH leading to $\mathrm{N}$-acetyl GSH (NAGSH) was performed using a reported procedure [57]. Deionized (DI) water with resistivity $>18 \mathrm{M} \Omega \cdot \mathrm{cm}$ 
was used for all experiments. The details of dansylation are given below.

\subsection{Synthesis of DGSH}

Synthesis of DGSH involves two steps [58]. The first step is the conjugation of the sulphonyl group of the dansyl moiety with the amino group of oxidized glutathione and the second step involves the breaking of the S-S bond resulting in DGSH. The synthetic route of the reaction is shown in Scheme 1 together with a photograph showing the fluorescence of an aqueous solution of DGSH under UV light. In brief, for $120 \mathrm{mg}$ of oxidized glutathione in $3 \mathrm{~mL}$ of DI-water ( $\mathrm{pH}$ of the solution adjusted to 8), 115 $\mathrm{mg}$ of $\mathrm{DnCl}$ in acetone was added dropwise and stirred well for $1 \mathrm{~h}$. After completion of the reaction, the product was washed thrice with chilled diethyl ether to remove the unreacted $\mathrm{DnCl}$. The solvent was removed using freeze drying and the compound was redissolved in $2 \mathrm{~mL}$ of tris-buffer ( $\mathrm{pH} 8$ ). To the above solution, $26 \mathrm{mg}$ of DTT in $2 \mathrm{~mL}$ tris buffer was added in a nitrogen atmosphere. The solution was stirred for $30 \mathrm{~min}$. After completion of the reaction, the $\mathrm{pH}$ of the solution was adjusted to 4 and it was freeze dried. The compound was purified by repeated washing with distilled methanol and finally with ethanol. The DGSH precipitate was dried and collected as a pale yellow powder. The geometry of DGSH was optimized by semi empirical Austin Model 1 (AM1)

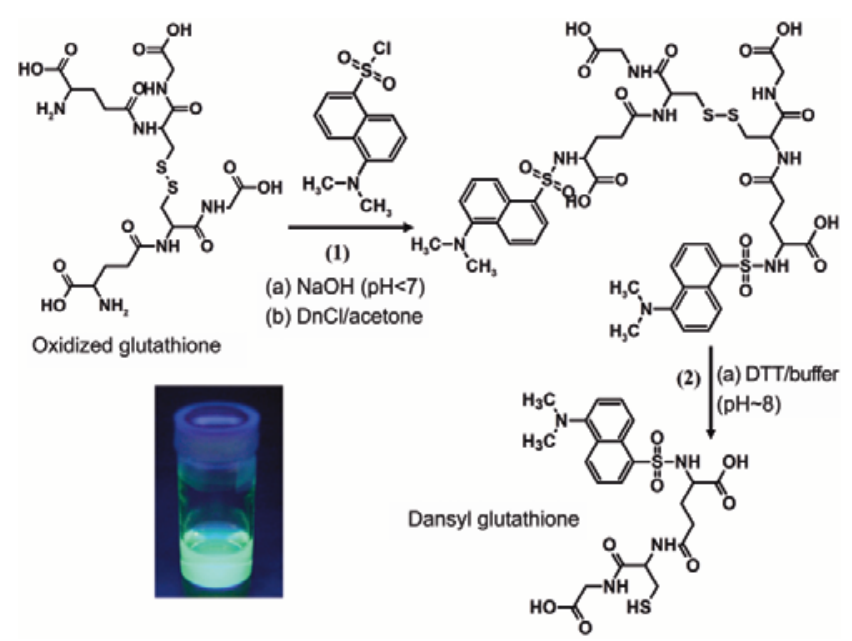

Scheme 1 Reaction sequence for the dansylation of glutathione with dansyl chloride $(\mathrm{DnCl})$. The photograph shows the fluorescence of a DGSH solution in water under UV light calculations using Gaussian-03 [59]. Details are given in the ESM (Fig. S-9).

\subsection{Synthesis of Au@SGAN/SGD}

The abbreviationAu@SGAN/SGD refers to Au nanoparticles protected with -SGAN and -SGD groups. The procedure used here for the synthesis of Au@SGAN/SGD follows the reported protocol [42] with a few modifications. To a $200 \mathrm{~mL}$ methanolic solution of $5 \mathrm{mmol} / \mathrm{L} \mathrm{HAuCl}_{4} \cdot 3 \mathrm{H}_{2} \mathrm{O}$, the ligands were added $(7 \mathrm{mmol} / \mathrm{L}$ of DGSH and $3 \mathrm{mmol} / \mathrm{L}$ of NAGSH were added to make a total ligand concentration of $10 \mathrm{mmol} / \mathrm{L}$ ). The mixture was cooled to $0^{\circ} \mathrm{C}$ in an ice bath for $30 \mathrm{~min}$. An aqueous solution of $\mathrm{NaBH}_{4}(0.2 \mathrm{~mol} / \mathrm{L}, 50 \mathrm{~mL})$, cooled to $0{ }^{\circ} \mathrm{C}$, was then injected rapidly into the above mixture under vigorous stirring. The mixture was allowed to react for another hour. The resulting precipitate was collected and washed repeatedly with methanol through centrifugal precipitation. Finally, the Au@SGAN/SGD precipitate was dried and collected as a dark brown powder. The supernatant of the centrifugation process was carefully collected and solvent was removed using vacuum and an aqueous solution of known volume was made. This solution was used for the calculation of the number of fluorophores present per nanoparticle using the extinction coefficient of the fluorophore. It was found that around 127 -SGD ligands were present on each nanoparticle.

\section{4 Synthesis of Au@SGAN/SGD superlattices}

The Au@SGAN/SGD superlattices were prepared by an established method [38-43]. It has previously been reported that $\mathrm{N}$-acetyl glutathione capped gold nanoparticles form stable superlattice crystals under acidic conditions [42]. About 30 mg Au@SGAN/SGD powder was redispersed in distilled water $(10 \mathrm{~mL})$ and the $\mathrm{pH}$ of the solution was adjusted by adding $12 \mathrm{~mol} / \mathrm{L} \mathrm{HCl}$ drop-wise. We optimised the $\mathrm{pH}$ by a trial and error method and found that high quality crystals are formed under highly acidic conditions of $\mathrm{pH}<1$, especially around $\mathrm{pH} 0.6-0.8$. The acidic nanoparticle solution was left undisturbed for a week, in the dark at a temperature below $25^{\circ} \mathrm{C}$. Typically we kept the sample solution in a styrofoam 
container. After storage for 7 days, crystallization took place at the air/water interface. A film with brown mirror-like appearance was seen indicating the formation of gold nanoparticle superlattices. The quality of the superlattice crystals was poor in the beginning stage of film formation and required a minimum of 2 to 3 weeks for the formation of quality crystals which gave a metallic appearance. This may be because the nanoparticles take time to diffuse at the growing interface to form an ordered assembly. The first step of superlattice formation is the creation of an island by the ordered arrangement of nanoparticles. The reason for the formation of epitaxial thin films at the interface can be attributed to the high tendency of heterogeneous nucleation at the interface. After the formation of superlattices, SEM measurements were carried out by transferring the film onto polished silicon wafers. Raman and confocal fluorescence measurements were carried out by transferring the film onto a thin glass plate. Film samples were transferred onto carbon coated copper grids and dried at room temperature for HRTEM measurements. The nanoparticle synthesis using mixed ligands and subsequent crystal formation is represented in Scheme 2.

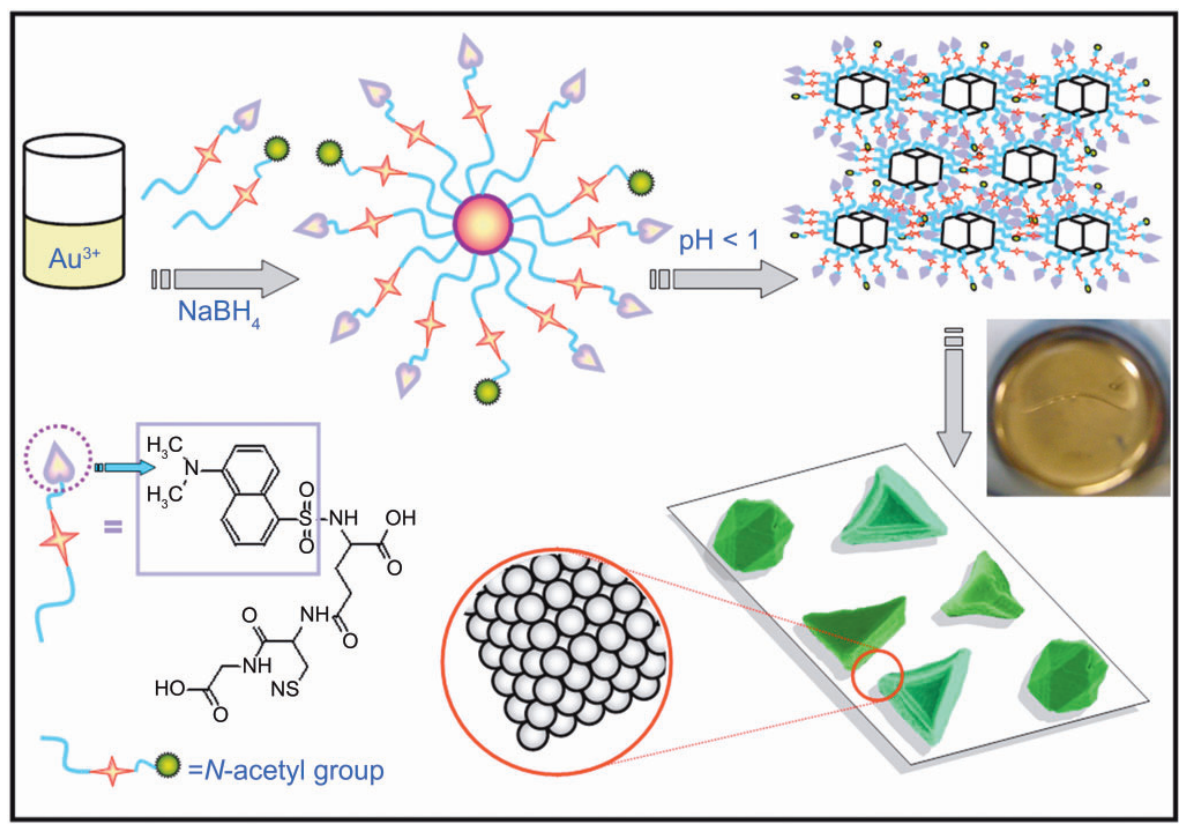

Scheme 2 Schematic representation of the nanoparticle synthesis and crystal formation. Different crystal morphologies are shown. The photograph shows the superlattice film formed at the interface. The picture is a view from the top of the beaker in which the superlattice film was grown. The nanoparticle solution below the film is black in color

\section{5 Methods}

UV-vis spectra were recorded using a Perkin Elmer Lambda 25 spectrometer. The UV/vis/NIR spectra of the crystals were recorded using a Varian $5 \mathrm{E}$ spectrometer in the range $200-2500 \mathrm{~nm} .{ }^{1} \mathrm{H}$ NMR spectra were recorded using a Bruker $400 \mathrm{MHz}$ instrument. The photoexcitation and fluorescence studies of the nanoparticle solutions were carried out using a Hitachi F-4500 spectrofluorimeter with a $100 \mathrm{~W}$ xenon lamp as the excitation source at a scan speed of $240 \mathrm{~nm} / \mathrm{s}$. Band pass for both excitation and emission monochromators was kept at $5 \mathrm{~nm}$. The Raman and fluorescence images were measured by using a Witec $\mathrm{GmbH}$ confocal Raman spectrometer equipped with a $514.5 \mathrm{~nm}$ Ar ion laser with a spot size $<1 \mu \mathrm{m}$. The laser had a maximum power of $40 \mathrm{~mW}$. The excitation laser was focused using a $100 \times$ objective and the signal was collected in a backscattering geometry and guided to a Peltier-cooled charge coupled device (CCD) detector. The sample was mounted on a piezo-equipped scan stage to enable spectral imaging. Single-spot spectra were also acquired using the same grating but with larger integration times. For improved resolution and to ascertain the peak positions, a grating with 1800 grooves / $\mathrm{mm}$ was also used while acquiring singlespot spectra. The effective scan range of the spectrometer was 0-9000 $\mathrm{cm}^{-1}$ (corresponding to a wavelength maximum of 958.2 $\mathrm{nm}$ for $514.5 \mathrm{~nm}$ excitation), with detection efficiency falling above $750 \mathrm{~nm}$. For spectral imaging, the desired area was partitioned into 10,000 squares (by means of an imaginary $100 \times 100$ matrix drawn over it), with each square representing a sampling point and consequently a pixel for the image. Typical signal acquisition time at each pixel of the image was $0.1 \mathrm{~s}$. The intensities of the desired portion of the spectra collected 
over all the pixels were compared by Scan CTRL Spectroscopy Plus Version 1.32 software, in order to construct a color-coded image. Spectral intensities acquired over a predefined area were automatically compared to generate color-coded images. In the images, regions coded yellow have maximum fluorescence intensities and regions shown in black have minimum signal intensities. Contact mode AFM measurements were carried out using a reflex coated Nanosensors silicon cantilever. The sample was mounted on a piezo-controlled scan stage. A diode laser of wavelength $980 \mathrm{~nm} \pm 5 \mathrm{~nm}$ was used as the beam deflector laser. The movement of the cantilever was detected using a segmented photodiode. HRTEM images were collected using JEOL 3010 UHR instrument. The superlattice films were lifted onto carbon coated copper grids and dried under ambient conditions. The sample was observed at $200 \mathrm{keV}$ to reduce electron beam induced damage. Mass spectrometric studies were conducted using an electrospray (ESI-MS) system, 3200 Q-TRAP LC/MS / MS (Applied Biosystems). Samples of $15 \mathrm{ppm}$ concentration taken in 1:1 water/methanol mixture were electrosprayed at 5 $\mathrm{kV}$. FESEM measurements were carried out using an FEI Nova NanoSEM 600 instrument. Regular SEM and EDAX were carried out with an FEI Quanta 200 instrument. Fluorescence images were recorded using a Leica SP-2 confocal microscope with a 458 nm laser. SAXS measurements were performed with a Bruker-AXS NanoSTAR instrument. The instrument has an $\mathrm{X}$-ray tube $(\mathrm{Cu} \mathrm{K} \alpha$ radiation, operated at $45 \mathrm{kV} / 35 \mathrm{~mA}$ ), cross-coupled Göbel mirrors, three-pinhole collimation, evacuated beam path, and a 2-D gas detector (HI-STAR) [60, 61]. The SAXS data were recorded in the $q$ range 0.007 to 0.22 $\AA^{-1}$ (i.e., $2 \theta=0.1^{\circ}$ to $3^{\circ}$ ).

\section{Conclusions}

We have fabricated fluorescent self-assembled 3-D nanoparticle superlattices at an air/water interface by adjusting the $\mathrm{pH}$ of the solution. The crystals so formed can be considered as "artificial solids", in the same way as nanoparticles and quantum dots are called artificial atoms [1]. A variety of tools have been used to characterize the nanoparticles as well as superlattices in great detail. The morphology of the crystals was analyzed by HRTEM and FESEM measurements. The stacking of the nanoparticles in the 3-D superlattices was characterized by SAXS analysis which shows that the constituent gold nanoparticles were stacked in an fcc pattern rather than hcp, with a particle-particle distance (centre-centre) of $10.5 \mathrm{~nm}$ as determined by the simulated peak positions. HRTEM also shows a cubic arrangement of nanoparticles. The FESM measurements showed the presence of superlattice crystals with different morphologies, including triangles, icosahedra, triangular pyramids, diamondlike structures, and so on. The fluorescence properties of the crystals were studied using confocal fluorescence imaging and confocal Raman microscopy. The fluorescence spectrum shows a broad emission peak with a red shift of $60 \mathrm{~nm}$, whereas nanoparticles in solution did not show any significant shift. This can be attributed to the strong electronic interactions between the fluorophore and the nanoparticle in the 3-D solids. The fluorescence images collected from the crystals were interpreted by comparing them with the FESEM images. From contact mode AFM, the average edge length and average thickness of a single triangle were calculated and were found to be around $7.5 \mu \mathrm{m} \pm 0.01 \mu \mathrm{m}$ and $0.54 \mu \mathrm{m} \pm 0.01 \mu \mathrm{m}$, respectively. We utilized the fluorescence properties of the dansyl glutathione superlattice crystals for the selective detection of BSA in the $\mathrm{nmol} / \mathrm{L}$ range in the solid state using the selective binding properties of the naphthalene ring with site I of BSA. The formation of such superlattices with functional nanoparticles presents several interesting possibilities for materials science. These systems can be considered as a new class of functional materials and it is possible to tune their functionality based on the requirements of a given application. This new class of functional materials can be used as an excellent platform on which to explore several interesting phenomena such as biomolecular detection, SERS, and gas sensing. 


\section{Acknowledgements}

The authors thank the Department of Science and Technology (DST), Government of India for constantly supporting our research program on nanomaterials. We gratefully thank Prof. C. N. R. Rao and Mr. Kanishka Biswas, JNCASR, Bangalore, India, for the SAXS measurements. Thanks are due to Prof. G. U. Kulkarni, Ms. T. Bhuvana and Ms. N. R. Selvi, JNCASR Bangalore, India, for the FESEM measurements. Thanks are due to Dr. C. Subramaniam for help in AFM measurements. We thank Mr. Mohammed Akbar Ali, Dept. of Chemistry, IIT Madras for the calculations using Gaussian-03. E. S. S. thanks the University Grants Commission (UGC) for a senior research fellowship.

Electronic Supplementary Material: Supplementary material is available in the online version of this article at http://dx.doi.org/10.1007/ s12274-009-9020-0 and is accessible free of charge.

\section{References}

[1] Collier, C. P.; Vossmeyer, T.; Heath, J. R. Nanocrystal superlattices. Annu. Rev. Phys. Chem. 1998, 49, 371404.

[2] Achermann, M.; Petruska, M. A.; Kos, S.; Smith, D. L.; Koleske, D. D.; Klimov, V. I. Energy-transfer pumping of semiconductor nanocrystals using an epitaxial quantum well. Nature 2004, 429, 642-646.

[3] Gur, I.; Fromer, N. A.; Geier, M. L.; Alivisatos, A. P. Airstable all-inorganic nanocrystal solar cells processed from solution. Science 2005, 310, 462-465.

[4] Maier, S. A.; Kik, P. G.; Atwater, H. A.; Meltzer, S.; Harel, E.; Koel, B. E.; Reguicha, A. A. G. Local detection of electromagnetic energy transport below the diffraction limit in metal nanoparticle plasmon waveguides. Nat. Mater. 2003, 2, 229-232.

[5] Hoinville, J.; Bewick, A.; Gleeson, D.; Jones, R.; Kasyutich, O.; Mayes, E.;Nartowski, A.; Warne, B.; Wiggins, J.; Wong, K. High density magnetic recording on protein-derived nanoparticles. J. Appl. Phys. 2003, 93, 7187-7189.

[6] Grunes, J.; Zhu, J.; Anderson, E. A.; Somorjai, G. A. Ethylene hydrogenation over platinum nanoparticle array model catalysts fabricated by electron beam lithography: Determination of active metal surface area. J. Phys. Chem. B 2002, 106, 11463-11468.

[7] Zayats, M.; Kharitonov, A. B.; Pogorelova, S. P.; Lioubashevski, O.; Katz, E.; Willner, I. Probing photoelectrochemical processes in $\mathrm{Au}-\mathrm{CdS}$ nanoparticle arrays by surface plasmon resonance: Application for the detection of acetylcholine esterase inhibitors. J. Am. Chem. Soc. 2003, 125, 16006-16014.

[8] Motesharei, K.; Myles, D. C. Molecular recognition on functionalized self-assembled monolayers of alkanethiols on gold. J. Am. Chem. Soc. 1998, 120, 7328-7336.

[9] Spinke, J.; Liley, M.; Guder, H. -J.; Angermaier, L.; Knoll, W. Molecular recognition at self-assembled monolayers: The construction of multicomponent multilayers. Langmuir 1993, 9, 1821-1825.

[10] Alivisatos, A. P.; Johnson, K. P.; Peng, X.; Wilson, T. E.; Loweth, C. J.; Bruchez, M. P.; Schultz, P. G. Organization of "nanocrystal molecules" using DNA. Nature 1996, 382, 609-611.

[11] Murray, C. B.; Kagan, C. R.; Bawendi, M. G. Selforganization of CdSe nanocrystallites into threedimensional quantum dot superlattices. Science 1995, 270, 1335-1338.

[12] Korgel, B. A.; Fitzmaurice, D. Self-assembly of silver nanocrystals into two-dimensional nanowire arrays. $A d v$. Mater. 1998, 10, 661-665.

[13] Wang, Z. L. Structural analysis of self-assembling nanocrystal superlattices. Adv. Mater. 1998, 10, 13-30.

[14] Harfenist, S. A.; Wang, Z. L.; Alvarez, M. M.; Vezmar, I.; Whetten, R. L. Highly oriented molecular Ag nanocrystal arrays. J. Phys. Chem. 1996, 100, 13904-13910.

[15] Sigman, M. B., Jr.; Saunders, A. E.; Korgel, B. A. Metal nanocrystal superlattice nucleation and growth. Langmuir 2004, 20, 978-983.

[16] Wang, Z. L.; Harfenist, S. A.; Vezmar, I.; Whetten, R. L.; Bentley, J.; Evans, N. D.; Alexander, K. B. Superlattices of self-assembled tetrahedral Ag nanocrystals. Adv. Mater. 1998, 10, 808-812.

[17] Kalsin, A. M.; Fialkowski, M.; Paszewski, M.; Smoukov, S. K.; Bishop, K. J. M.; Grzybowski, B. A. Electrostatic selfassembly of binary nanoparticle crystals with a diamondlike lattice. Science 2006, 312, 420-424.

[18] Harfenist, S. A.; Wang, Z. L.; Whetten, R. L.; Vezmar, I.; Alvarez, M. M. Three-dimensional hexagonal closepacked superlattice of passivated Ag nanocrystals. Adv. 
Mater. 1997, 9, 817-822.

[19] Murray, C. B.; Kagan, C. R.; Bawendi, M. G. Synthesis and characterization of monodisperse nanocrystals and close-packed nanocrystal assemblies. Annu. Rev. Mater. Sci. 2000, 30, 545-610.

[20] Ohara, P. C.; Heath, J. R.; Gelbart, W. M. Self-assembly of submicrometer rings of particles from solutions of nanoparticles. Angew. Chem. Int. Ed. Engl. 1997, 36, 1078-1080.

[21] Shevchenko, E. V.; Talapin, D. V.; Kotov, N. A.; O’Brien, S.; Murray, C. B. Structural diversity in binary nanoparticle superlattices. Nature 2006, 439, 55-59.

[22] Taleb, A.; Petit, C.; Pileni, M. P. Synthesis of highly monodisperse silver nanoparticles from AOT reverse micelles: A way to 2-D and 3-D self-organization. Chem. Mater. 1997, 9, 950-959.

[23] Daniel, M. -C.; Astruc, D. Gold nanoparticles: Assembly, supramolecular chemistry, quantum-size-related properties, and applications toward biology, catalysis, and nanotechnology. Chem. Rev. 2004, 104, 293-346.

[24] Rogach, A. L.; Talapin, D. V.; Shevchenko, E. V.; Kornowski, A.; Haase, M.; Weller, H. Organization of matter on different size scales: Monodisperse nanocrystals and their superstructures. Adv. Funct. Mater. 2002, 12, 653-664.

[25] Redl, F. X.; Cho, K. S.; Murray, C. B.; O'Brien, S. Three-dimensional binary superlattices of magnetic nanocrystals and semiconductor quantum dots. Nature 2003, 423, 968-971.

[26] Stoeva, S. I.; Prasad, B. L. V.; Uma, S.; Stoimenov, P. K.; Zaikovski, V.; Sorensen, C. M.; Klabunde, K. J. Facecentered cubic and hexagonal closed-packed nanocrystal superlattices of gold nanoparticles prepared by different methods. J. Phys. Chem. B 2003, 107, 7441-7448.

[27] Dabbousi, B. O.; Murray, C. B.; Rubner, M. F.; Bawendi, M. G. Langmuir-Blodgett manipulation of size-selected CdSe nanocrystallites. Chem. Mater. 1994, 6, 216-219.

[28] Pileni, M. P. Nanocrystal self-assemblies: Fabrication and collective properties. J. Phys. Chem. B 2001, 105, 33583371.

[29] Stoeva, S.; Klabunde, K. J.; Sorensen, C. M.; Dragieva, I. Gram-scale synthesis of monodisperse gold colloids by the solvated metal atom dispersion method and digestive ripening and their organization into two- and three-dimensional structures. J. Am. Chem. Soc. 2002, 124, 2305-2311.
[30] Binder, W. H. Supramolecular assembly of nanoparticles at liquid-liquid interfaces. Angew. Chem. Int. Ed. 2005, 44, 5172-5175.

[31] Sanyal, M. K.; Agrawal, V. V.; Bera, M. K.; Kalyanikutty, K. P.; Daillant, J.; Blot, C.; Kubowicz, S.; Konovalov, O.; Rao. C. N. R. Formation and ordering of gold nanoparticles at the toluene-water interface. J. Phys. Chem. C. 2008, $112,1739-1743$, and the reference, $6 a$ and $6 \mathrm{~b}$ cited therein.

[32] Sarathy, K. V.; Kulkarni, G. U.; Rao, C. N. R. A novel method of preparing thiol-derivatised nanoparticles of gold, platinum and silver forming superstructures. Chem. Commun. 1997, 537-538.

[33] Lin, Y.; Skaff, H.; Emrick, T.; Dinsmore, A. D.; Russell, T. P. Nanoparticle assembly and transport at liquid-liquid interfaces. Science 2003, 299, 226-229.

[34] Talapin, D. V.; Shevchenko, E. V.; Kornowski, A.; Gaponik, N.; Haase, M.; Rogach, A. L.; Weller, H. A new approach to crystallization of CdSe nanoparticles into ordered three-dimensional superlattices. Adv. Mater. 2001, 13, 1868-1871.

[35] Boal, A. K.; Ilhan, F.; DeRouchey, J. E.; Thurn-Albrecht, T.; Russel, T. P.; Rotello, V. M. Self-assembly of nanoparticles into structured spherical and network aggregates. Nature 2000, 404, 746-748.

[36] Demer, L. M.; Ginger, D. S.; Park, S. -J.; Li, Z.; Chung, S. -W.; Mirkin, C. A. Direct pattering of modified oligonucleotides on metals and insulators by dip-pen nanolithography. Science 2002, 296, 1836-1838.

[37] Sanyal, A.; Norsten, T. B.; Uzun, O.; Rotello, V. M. Adsorption/desorption of mono- and di-block copolymers on surfaces using specific hydrogen bonding interactions. Langmuir 2004, 20, 5958-5964.

[38] Kimura, K.; Sato, S.; Yao, H. Particle crystals of surface modified gold nanoparticles growth from water. Chem. Lett. 2001, 30, 372-373.

[39] Wang, S. H.; Sato, S.; Kimura, K. Preparation of hexagonal-close-packed colloidal crystals of hydrophilic monodisperse gold nanoparticles in bulk aqueous solution. Chem. Mater. 2003, 15, 2445-2448.

[40] Wang, S. H.; Yao, H.; Sato, S.; Kimura, K. Inclusionwater-cluster in a three-dimensional superlattice of gold nanoparticles. J. Am. Chem. Soc. 2004, 126, 74387439.

[41] Yang, Y.; Liu, S.; Kimura, K. Superlattice formation from polydisperse Ag nanoparticles by a vapor-diffusion 
method. Angew. Chem. Int. Ed. 2006, 45, 5662-5665.

[42] Yao, H.; Minami, T.; Hori, A.; Koma, M.; Kimura, $K$. Fivefold symmetry in superlattices of monolayerprotected gold nanoparticles. J. Phys. Chem B. 2006, 110, 14040-14045.

[43] Nishida, N.; Shibu, E. S.; Yao, H.; Oonishi, T.; Kimura, K.; Pradeep, T. Fluorescent gold nanoparticle superlattices. Adv. Mater. 2008, 20, 4719-4723.

[44] Kiely, C. J.; Fink, J.; Brust, M.; Bethell, D.; Schiffrin, D. J. Spontaneous ordering of bimodal ensembles of nanoscopic gold clusters. Nature 1998, 396, 444-446.

[45] Rogach, A. L. Binary superlattices of nanoparticles: Selfassembly leads to "metamaterials". Angew. Chem. Int. Ed. 2004, 43, 148-149.

[46] Urban, J. J.; Talapin, D. V.; Shevchenko, E. V.; Kagan, C. R.; Murray, C. B. Synergistic effects in binary nanocrystal superlattices: Enhanced p-type conductivity in selfassembled PbTe/Ag2Te thin films. Nat. Mater. 2007, 6, 115-121.

[47] Chen, Z. Y.; Moore, J.; Radtke, G.; Sirringhaus, H.; O'Brien, S. Binary nanoparticle superlattices in the semiconductor-semiconductor system: CdTe and CdSe. J. Am. Chem. Soc. 2007, 129, 15702-15709.

[48] Chen, Z.; O’Brien, S. Structure direction of II-VI semiconductor quantum dot binary nanoparticle superlattices by tuning radius ratio. ACS Nano 2008, 2, 1219-1229.

[49] Terrill, R. H.; Postlethwaite, T. A. P.; Chen, C. H.; Poon, C. D; Terzis, A.; Chen, A.; Hutchison, J. E.; Clark, M. R.; Wignall, G. Monolayers in three dimensions: NMR, SAXS, thermal, and electron hopping studies of alkanethiol stabilized gold clusters. J. Am. Chem. Soc. 1995, 117, 12537-12548.

[50] Shibu, E. S.; Habeeb Muhammed, M. A.; Tsukuda, T.; Pradeep, T. Ligand exchange of $\mathrm{Au}_{25} \mathrm{SG}_{18}$ leading to functionalized gold clusters: Spectroscopy, kinetics and luminescence. J. Phys. Chem C. 2008 112, 1216812176.

[51] Habeeb Muhammed, M. A.; Shaw, A. K.; Pal, S. K.; Pradeep, T. Quantum clusters of gold exhibiting FRET. J. Phys. Chem C. 2008, 112, 14324-14330.

[52] Wang, Z. L. Transmission electron microscopy of shapecontrolled nanocrystals and their assemblies. J. Phys.
Chem. B 2000, 104, 1153-1175.

[53] Wang, Z. L.; Harfenist, A. S.; Whetten, R. L.; Bentley, J.; Evans, N. D. Bundling interdigitation of adsorbed thiolate groups in self-assembled nanocrystal superlattices. J. Phys. Chem. B 1998, 102, 3068-3072.

[54] Maxwell, D. J.; Taylor, J. R.; Nie, S. Self-assembled nanoparticle probes for recognition and detection of biomolecules. J. Am. Chem. Soc. 2002, 124, 96069612.

[55] Pandey, R. K.; Constantine, S.; Tsuchida, T.; Zheng, G.; Medforth, C. J.; Aoudia, M.; kozyrev, A. N.; Rodgers, M. A. J.; Kato, H.; Smith, K. M.; Dougherty, T. J. Synthesis, photophysical properties, in vivo photosensitizing efficacy, and human serum albumin binding properties of some novel bacteriochlorins. J. Med. Chem. 1997, 40, 2770-2779.

[56] Chang, C. C.; Wu, H. L.; Kuo, C. H.; Huang, M. H. Hydrothermal synthesis of monodispersed octahedral gold nanocrystals with five different size ranges and their self-assembled structures. Chem. Mater. 2008, 20, 7570-7574.

[57] Levy, E. J.; Anderson, M. E.; Meister, A. On the synthesis and characterization of $\mathrm{N}$-formyl glutathione and $\mathrm{N}$-acetyl glutathione. Anal. Biochem. 1993, 214, 135137.

[58] Gan, J. P.; Harper, T. W.; Hsueh, M. -M.; Qu, Q. L.; Humphreys, W. G. Dansyl glutathione as a trapping agent for the quantitative estimation and identification of reactive metabolites. Chem. Res. Toxicol. 2005, 18, 896-903.

[59] Frisch, M. J.; Trucks, G. W.; Schlegel, H. B.; Scuseria, G. E.; Robb, M. A.; Cheeseman, J. R.; Montgomery, J. A., Jr.; Vreven, T.; Kudin, K. N. Gaussian 03, Revision C.02, Gaussian, Inc., Wallingford CT, 2004.

[60] Biswas, K.; Varghese, N.; Rao, C. N. R. Growth kinetics of gold nanocrystals: A combined small-angle X-ray scattering and calorimetric study. Small 2008, 4, 649655.

[61] Pedersen, J. S. A flux- and background- optimized version of the nanoSTAR small-angle $X$-ray scattering camera for solution scattering. J. Appl. Crystallogr. 2004, $37,369-380$ 